\title{
Influencing Factors of 2D Shear Wave Elastography of the Muscle - An Ex Vivo Animal Study
}

(1) $\odot \Theta$

Authors

Marga B Rominger ${ }^{1}$, Pascal Kälin ${ }^{1}$, Monika Mastalerz ${ }^{1}$, Katharina Martini ${ }^{1}$, Volker Klingmüller ${ }^{1}$, Sergio Sanabria ${ }^{2}$, Thomas Frauenfelder ${ }^{1}$

Affiliations

1 Institute of Diagnostic and Interventional Radiology, University Hospital Zurich, University of Zurich, Zurich, Switzerland

2 Eidgenossische Technische Hochschule Zurich, Computer Vision Laboratory, Zurich, Switzerland

Key words

muscular, soft tissues, ultrasound, ultrasound 2D

received 22.12 .2017

revised 27.03.2018

accepted 29.03.2018

Bibliography

DOI https://doi.org/10.1055/a-0619-6058

Ultrasound Int Open 2018; 4: E54-E60

(c) Georg Thieme Verlag KG Stuttgart · New York

ISSN 2199-7152

Correspondence

Prof. Marga B Rominger, MD

Institute of Diagnostic and Interventional Radiology

UniversitätsSpital Zurich

Rämistrasse 100, Zürich

8006, Switzerland

Tel.: + 41/442/552900

Marga.Rominger@usz.ch

\section{ABSTRACT}

Objective To evaluate measurement confounders on 2D shear wave elastography (2D-SWE) elastography of muscle.

Materials and Methods Ex vivo, porcine muscle was examined with a GE LOGIQ E9 ultrasound machine with a $9 \mathrm{~L}$ linear $(9 \mathrm{MHz})$ and C1-6 convex probe (operating at 2.5 or $6 \mathrm{MHz}$ ). The influence of different confounders on mean shear wave velocity (SWVmean) was analyzed: probes, pressure applied by probe, muscle orientation, together with the impact of different machine settings such as frequency, placement depth and size of region of interest (ROI). The mean of twelve repeated SWVmean measurements $(\mathrm{m} / \mathrm{s})$ and coefficient of variation (CV; standard deviation/mean in \%) were assessed for each test configuration.

Results Reproducibility (CV) and maximum possible tissue depth of the linear probe were inferior to the convex probe. With the linear probe, there was a linear decrease of SWVmean with placement depth from $4.56 \mathrm{~m} / \mathrm{s}$ to $1.81 \mathrm{~m} / \mathrm{s}$. A significant increase of SWVmean $(p<0.001)$ was observed for larger ROI widths (range $3.96 \mathrm{~m} / \mathrm{s}$ to $6.8 \mathrm{~m} / \mathrm{s}$ ). A change in the machine operation mode ('penetration' instead of 'general') led to a significant increase of SWVmean ( $p=0.04)$. SWVmean in the longitudinal direction of muscle was significantly higher than in cross section $(p<0.001)$ (e. g. $4.56 \mathrm{~m} / \mathrm{s}$ versus $3.42 \mathrm{~m} / \mathrm{s}$ ). An increase of linear probe pressure significantly increased muscle SWVmean from $5.29 \mathrm{~m} / \mathrm{s}$ to $7.21 \mathrm{~m} / \mathrm{s}(\mathrm{p}<0.001)$.

Conclusions 2D-SWE of muscle is influenced by a wealth of parameters. Therefore, standardization of measurement is advisable before application in clinical research studies and routine patient assessment.

\section{Introduction}

Ultrasound elastography is widely used [1-3] and ultrasound elastography of muscles is a rapidly emerging field with many applications for focal and systemic disease [4]. Increased collagen content and stiffness are seen in numerous musculoskeletal pathologies, including spasticity, as well as aging (sarcopenia). One of the challenges of muscle ultrasound is anisotropy, where the ultrasound beam may be reflected in a different direction than the excited ultrasound beam, especially if it is excited with an inclination with respect to the muscle fibers. Due to the skewing of ultrasound energy along the muscle fibers, some elastography signals may be lost [5].

2D shear wave elastography (2D-SWE) is a newer method for assessing tissue stiffness. 2D-SWE is a quantitative measurement that displays a color-coded shear wave velocity (SWV) map overlying the greyscale B-mode image within a selected region of interest (ROI) of variable size. Then, a circular ROI of variable size is positioned by the sonographer in the area of interest within the color-coded map. The average SWV (SWVmean) is calculated by 
the device from the SWV value of each pixel included within the circular ROI.

Non-linear sound characteristics of tissue continue to be a major confounder of elastography, potentially yielding different measurements under different probe and operator tissue manipulations, thus preventing a repeatable and hence reliable diagnosis [6]. Technical and instrument-related as well as biologic and patient-related factors constitute confounders of stiffness measurements [7].

Confounders have been mainly studied for acoustic radiation force impulse (ARFI) working with a fixed, predefined ROI sample size, the convex probe and the liver organ enclosed in the rib cage [7-11]. Thus, the influence of confounders on 2D-SWE muscle measurements may differ from those of ARFI and liver assessment.

The aim of the ex vivo study was to assess possible measurement confounders ( $\triangleright$ Table 1), e. g, linear versus convex probe, probe pressure and ROI placement and size, on 2D-SWE of porcine muscle.

\section{Materials and Methods}

\section{Experimental setting}

We examined fresh porcine muscle from the slaughterhouse placed in a plastic box (Tupperware) within twelve hours after explantation at room temperature. The box had a self-made small opening in the cover to accommodate the probes. Setup parameters (probes, probe pressure, and muscle orientation), machine settings (frequencies, placement depths, B-mode and circular ROI sizes), and manufacturer's 'general' and 'penetration' presets were studied at a defined sample location ( $\triangleright$ Table 1 ). To avoid operator-dependent motion and pressure uncertainties, the probes were mounted on a fixed rack. To examine the influence of probe pressure on 2D-SWE, measurements with a digital weighting scale beneath the containing box were performed ( $\mathbf{F i g} \mathbf{1}$ ).

\section{Ultrasound measurements}

2D SWE examinations were performed with a $9 \mathrm{~L}$ linear probe and a C1-6 convex probe. SWE reconstruction was performed with a LOGIQ E9 system (Software Version R5, Revision 1.1; GE Healthcare, Wauwatosa, WI), which combined Comb-push Ultrasound Shear Elastography together with Time Aligned Sequential Tracking [12]. All measurements were performed with the manufacturer standard preset: 'general'. In addition, we measured muscle with the manufacturer alternative preset 'penetration'.

Following the manufacturer's manual and our clinical study protocol, we selected a rectangular (linear probe) or trapezoid (convex probe) ROI on the conventional B-mode ultrasound screen. We picked a homogeneous SWV map showing stability for 2-4 images. Thereafter, a circular ROI encompassing the entire SWV map was placed. The SWV values were expressed in meters per second $(\mathrm{m} / \mathrm{s})$ representing the average of color-coded pixels within the circular ROI (SWVmean). In each location and for each variable, twelve 2D-SWV measurements were performed. Care was taken not to include heterogeneities in the ROI, such as large connective tissue fibers in the striate muscle.

\section{Influence of probe, frequency, ROI placement depth and size and machine operation presets}

All ROI placement depth measurements were performed with the linear probe $(9 \mathrm{MHz})$ and convex probe ( 2.5 and $6 \mathrm{MHz})$. We tested the convex probe in addition to the normally used linear probe for deep-lying muscles or possible large patients. The first B-mode ROI

- Table 1 Porcine muscle.

\begin{tabular}{|c|c|c|c|c|c|c|c|c|c|c|}
\hline Probe & \multicolumn{4}{|c|}{ Linear } & \multicolumn{6}{|c|}{ Convex } \\
\hline Frequency & \multicolumn{4}{|c|}{$9 \mathrm{MHz}$} & \multicolumn{4}{|c|}{$2.5 \mathrm{MHz}$} & \multirow{2}{*}{\multicolumn{2}{|c|}{$\begin{array}{c}6 \mathrm{MHz} \\
\text { Longitudinal }\end{array}$}} \\
\hline Fiber & \multicolumn{2}{|c|}{ Longitudinal } & \multicolumn{2}{|c|}{ Cross-section } & \multicolumn{2}{|c|}{ Longitudinal } & \multicolumn{2}{|c|}{ Cross-section } & & \\
\hline $\begin{array}{l}\text { Insertion } \\
\text { depth }(\mathrm{cm})\end{array}$ & $\mathrm{sWV}(\mathrm{m} / \mathrm{s})$ & CV & $\mathrm{SWV}(\mathrm{m} / \mathrm{s})$ & CV & $\mathrm{SWV}(\mathrm{m} / \mathrm{s})$ & $C V$ & $\mathrm{sWV}(\mathrm{m} / \mathrm{s})$ & CV & $\operatorname{SWV}(\mathrm{m} / \mathrm{s})$ & $\mathrm{CV}$ \\
\hline 0 & n.m. & n.m. & n.m. & n.m. & 1.89 & $8 \%$ & 1.75 & $16 \%$ & $1.60^{\S}$ & $15 \%$ \\
\hline 1 & 4.56 & $1 \%$ & $3.42^{\circ}$ & $1 \%$ & 2.17 & $7 \%$ & $1.87^{\circ}$ & $13 \%$ & 2.18 & $13 \%$ \\
\hline 2 & 3.68 & $3 \%$ & $3.02^{\circ}$ & $8 \%$ & 2.67 & $5 \%$ & 2.47 & $11 \%$ & $2.55^{\S}$ & $4 \%$ \\
\hline 3 & 2.48 & $8 \%$ & $1.99^{\circ}$ & $28 \%$ & 2.74 & $5 \%$ & 2.75 & $18 \%$ & 2.81 & $8 \%$ \\
\hline 4 & $1.81^{*}$ & $34 \%$ & $1.34^{*}$ & $19 \%$ & 2.35 & $5 \%$ & $2.60^{\circ}$ & $6 \%$ & 2.31 & $9 \%$ \\
\hline 5 & n.m. & n.m. & n.m. & n.m. & 2.22 & $4 \%$ & $2.55^{\circ}$ & $5 \%$ & 2.24 & $13 \%$ \\
\hline 6 & n.m. & n.m. & n.m. & n.m. & 1.91 & $103 \%$ & 2.04 & $11 \%$ & 1.95 & $19 \%$ \\
\hline Mean & $3.13^{*}$ & & $2.44^{* \circ}$ & & 2.28 & & 2.29 & & 2.23 & \\
\hline
\end{tabular}

Mean shear wave velocities (SWV) and coefficient of variation (CV) at different insertion depths, probes, probe frequencies and fiber orientations. n.m. = measurement failed. ${ }^{*}=$ mean includes misfiring with less than twelve, but six or more successful measurements. ${ }^{\circ}=$ significant mean difference of longitudinal and cross-section fiber directions. $\S=$ significant mean difference of convex probe operating at $2.5 \mathrm{MHz}$ vs. $6 \mathrm{MHz}$ 
with a height of one $\mathrm{cm}$ was placed directly under the surface of the muscle. The next ROI was placed with its upper border corresponding to the lower border of the previous ROI. Step-by-step SWV measurements were performed with an increasing distance of one $\mathrm{cm}$ between the probe and ROI until SWV measurements reached the lower end or completely failed for the twelve measurements at two consecutive placement depths in a row. To evaluate the influence of the size of the circular ROI, we increased the circular ROI diameter from 0.6 to $3 \mathrm{~cm}$ starting at the center of a $1 \mathrm{~cm} x$ $1 \mathrm{~cm} \mathrm{~B}$-mode ROI until the rectangular B-mode ROI was encompassed.

In addition, we measured four adjacent $1 \mathrm{~cm} \times 1 \mathrm{~cm}$ square B-mode ROls, corresponding to four quadrants (upper left, upper right, lower left and lower right) starting at a depth of $1 \mathrm{~cm}$ and in parallel to the muscle fibers. To test the influence of ROI width, a $2 \mathrm{~cm} \times 1 \mathrm{~cm}$ upper and lower row ROI (horizontal rectangular), $1 \mathrm{~cm}$ $x 2 \mathrm{~cm}$ left- and right-sided ROI (vertical rectangular), as well as $2 \mathrm{~cm} \times 2 \mathrm{~cm}$ ROI encompassing all four quadrants were measured at the same location ( $\triangleright$ Fig. 2 ). The ROIs were measured with both the manufacturer's 'general' and 'penetration' presets. When the "penetration" preset is $\mathrm{ON}$, the push pulse duration is longer. The amplitude of the shear wave generated will be higher, leading to an increase in penetration and signal-to-noise ratio. The trade-off with the "penetration" preset is a lower frame rate [13].

\section{Influence of muscle fiber orientation}

To assess the effect of muscle fiber orientation of porcine muscle in SWV, we measured in both the longitudinal (parallel) direction and the cross-section (vertical) with respect to the orientation of the muscle fibers. In addition, the muscle was tilted by $180^{\circ}$ (flipped) to scan the longitudinal direction of the muscle from the contralateral side to test for tissue inhomogeneity or accumulation due to bedding within the box.

\section{Influence of increasing probe pressure}

Increasing probe pressure from zero to $3000 \mathrm{~g}$ scale weight was manually applied to the muscle surface in the longitudinal direction (surface area of the probe $5 \mathrm{~cm} \times 1 \mathrm{~cm}$ ). The ROI of $1 \mathrm{~cm} \times 1 \mathrm{~cm}$ was placed with its upper border $1 \mathrm{~cm}$ below the muscle surface.

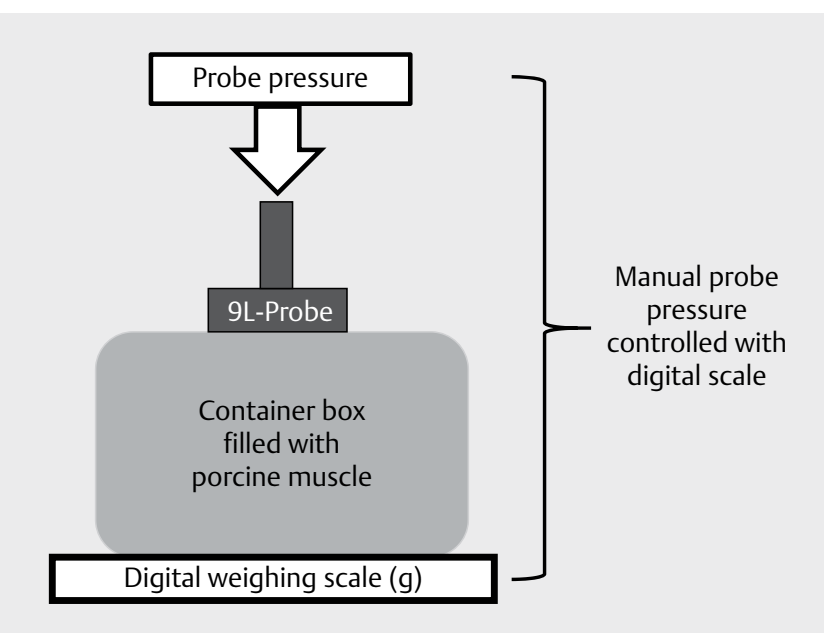

- Fig. 1 Schematic drawing of probe pressure increase.

\section{Statistical analysis}

IBM SPSS Statistics software (version 2010) and Microsoft Excel 2011 were used for statistical analysis. The complete feasibility of the measurement was defined as the success of 12 consecutive measurements without misfiring. The reproducibility of 2D-SWE was estimated with the percentage ratio of SD to the mean of the repeated tissue SWV measurements (coefficient of variation, CV) [14]. A CV ratio of $10 \%$ and below was considered to be a reliable measurement [15-17]. CVs above $10 \%$ and/or incomplete feasibility of 12 measurements in a row were considered to be unreliable measurements. Statistical differences were assessed using analysis of variance (ANOVA), Student's t-test and/or linear regression analysis. $P<0.05$ was considered statistically significant. When applicable, Bonferroni correction for multiple comparisons was applied.

\section{Results}

\section{Influence of probe, frequency, ROI placement depth and size and machine operation presets}

The SWVmean and CV of the linear and convex probe at different placement depths within porcine muscle are shown in $>$ Table 2. With the linear probe SWV measurements immediately below the surface were not feasible. Measurements were feasible at a placement depth between 1 and $3 \mathrm{~cm}$, incomplete (less than twelve but more than six successful measurements) at a placement depth of $4 \mathrm{~cm}$ and not feasible at a placement depth greater than $5 \mathrm{~cm}$. There was a linear decrease of the linear probe's SWVmean values at a placement depth from $1 \mathrm{~cm}$ to $4 \mathrm{~cm}(p<0.001)$ for both longitudinal and transverse muscle fiber orientation. Using the convex probe, SWVmean measurements were measurable for the whole

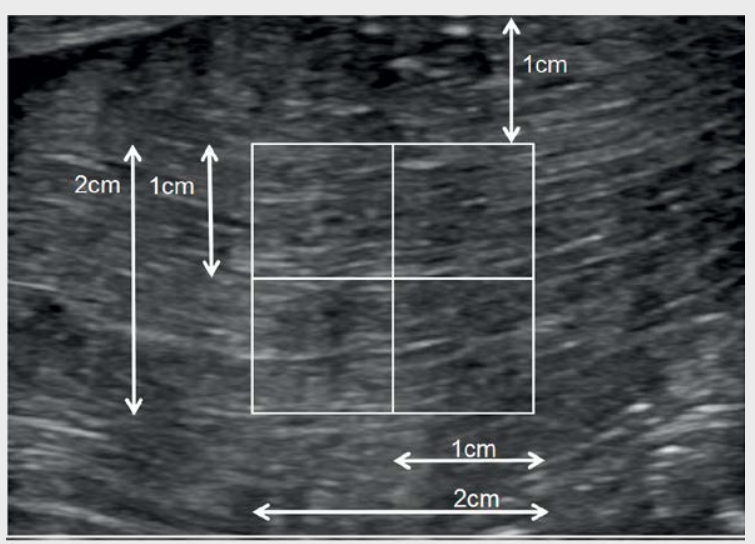

a.

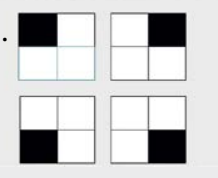

b.

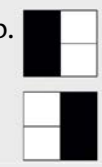

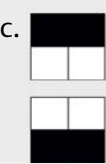

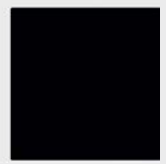

- Fig. 2 Schematic drawing of different B-mode ROIs (black-shaded background), as used for $>$ Table 3 and shown in $>$ Fig. 3: a. $1 \mathrm{~cm} x$ $1 \mathrm{~cm}$ left upper, left lower, right upper, and right lower square; $\mathbf{b}$. $1 \mathrm{~cm} \times 2 \mathrm{~cm}$ left-sided and right-sided rectangles; $\mathrm{c} .2 \mathrm{~cm} \times 1 \mathrm{~cm}$ upper and lower row rectangle; $\mathbf{d}$. $2 \mathrm{~cm} \times 2 \mathrm{~cm}$ square. 
depth of muscle for both test frequencies. SWV values immediately under the muscle surface were lower than values at deeper placement. The difference between SWVmean immediately under the muscle surface and SMVmean at a deeper placement reached statistical significance for depths within 2 to $5 \mathrm{~cm}$ at $2.5 \mathrm{MHz}$ and for all depths of 1 to $6 \mathrm{~cm}$ at $6 \mathrm{MHz}(\mathrm{p}<0.05)$.

- Table 3 and $>$ Fig. 3 show the dependency of SWVmean and $\mathrm{CV}$ on ROI placement depth and width using the $9 \mathrm{~L}$ linear probe. There was a significantly decreased SWVmean $(p<0.001)$ for the lower ROI squares and rectangles compared to the upper ones, as well as a significantly increased SWVmean $(p<0.001)$ for a width of $2 \mathrm{~cm}$ compared to $1 \mathrm{~cm}$.

'Penetration' preset mode led to a significant overall increase in SWVmean $(p=0.04)$, and in particular for the lower rectangular ROI quadrants and rectangles compared to the 'general' mode $(p<0.001)$. ( $>$ Table 3$)$

An increase in circular, color-coded ROI showed a minor variation in SWVmean $(5.42-5.21 \mathrm{~m} / \mathrm{s})$ and constant CV of $2 \%$.

\section{Influence of muscle fiber orientation}

With the $9 \mathrm{~L}$ linear probe the SWVmean in the longitudinal direction of muscle was significantly higher than in the cross-section ( 3.13 vs. $2.46 \mathrm{~m} / \mathrm{s}, \mathrm{p}<0.001$ ) ( $\triangleright$ Table 2 ). With the $C 1-6$ convex probe $(2.5 \mathrm{MHz})$, there was no significant overall difference between SWVmean in the longitudinal direction and in the cross-section ( 2.28 vs. $2.29 \mathrm{~m} / \mathrm{s} ; \mathrm{p}=0.86$ ). Comparing SWVmean with the C1-6 convex probe (2.5 MHz) in the longitudinal direction from an- terior/posterior with posterior/anterior measurements did not show a significant difference ( 2.28 vs. $2.37 \mathrm{~m} / \mathrm{s} ; \mathrm{p}=0.156$ ).

\section{Influence of increasing probe pressure}

Manual increase of probe pressure with the $9 \mathrm{~L}$ linear probe in the longitudinal direction with respect to the muscle fibers resulted in an increase in SWVmean from $5.29 \mathrm{~m} / \mathrm{s}$ to $7.21 \mathrm{~m} / \mathrm{s}$, which was most pronounced between no pressure and a scale weight of $500 \mathrm{~g}$ $(p<0.001)$. (> Fig. 4)

\section{Discussion}

In this ex vivo animal study, we found a significant dependency of 2D-SWE on ROI placement depth and width, preset mode, muscle fiber orientation, as well as probe pressure for muscle elastography. The SWVmean values of the linear probe were different from the values measured with the convex probe ( $\triangleright$ Table $\mathbf{1}$ ).

SWVmean measurements may be influenced by: a) the excitation sequence (acoustic-radiation force pushes), b) the imaging sequence - ultrafast plane wave, c) tissue displacement tracking algorithms, d) shear wave speed estimation approach [12].

Using the linear probe in our ex vivo model, we found a substantial and significant linear decay of SWVmean in muscle with increasing ROI placement depth. With the convex probe, we did not find any significant influence of ROI placement depth on SWV measurements except for very superficial and deep muscle areas. Ewertsen et al. [5] found that SWV in healthy muscle decreased with increas-

- Table 2 Bovine liver.

\begin{tabular}{|c|c|c|c|c|c|c|}
\hline \multirow{3}{*}{$\begin{array}{l}\text { Probe } \\
\text { Frequency } \\
\text { Insertion depth }(\mathrm{cm})\end{array}$} & \multicolumn{2}{|c|}{ Linear } & \multicolumn{4}{|c|}{ Convex } \\
\hline & \multicolumn{2}{|c|}{$9 \mathrm{MHz}$} & \multicolumn{2}{|c|}{$2.5 \mathrm{MHz}$} & \multicolumn{2}{|c|}{$6 \mathrm{MHz}$} \\
\hline & $\operatorname{SWV}(\mathrm{m} / \mathrm{s})$ & CV & $\operatorname{sWV}(\mathrm{m} / \mathrm{s})$ & CV & $\operatorname{sWV}(\mathrm{m} / \mathrm{s})$ & CV \\
\hline 0 & 1.32 & $1 \%$ & 1.16 & $1 \%$ & 1.27 & $1 \%$ \\
\hline 1 & 1.53 & $1 \%$ & 1.32 & $1 \%$ & 1.33 & $2 \%$ \\
\hline 2 & 2.03 & $21 \%$ & 1.26 & $5 \%$ & 1.24 & $1 \%$ \\
\hline 3 & 1.59 & $48 \%$ & 1.15 & $1 \%$ & 1.37 & $0 \%$ \\
\hline 4 & $1.06^{*}$ & $25 \%$ & 1.17 & $3 \%$ & 1.40 & $10 \%$ \\
\hline 5 & $1.41^{*}$ & $61 \%$ & 1.15 & $1 \%$ & 1.26 & $1 \%$ \\
\hline 6 & $1.26^{*}$ & $31 \%$ & 1.32 & $1 \%$ & 1.27 & $3 \%$ \\
\hline 7 & n.m. & n.m. & 1.27 & $1 \%$ & 1.28 & $1 \%$ \\
\hline 8 & n.m. & n.m. & 1.53 & $3 \%$ & 1.38 & $3 \%$ \\
\hline 9 & n.m. & n.m. & 1.84 & $18 \%$ & 1.36 & $6 \%$ \\
\hline 10 & n.m. & n.m. & 2.38 & $46 \%$ & 1.76 & $13 \%$ \\
\hline 11 & n.m. & n.m. & n.m. & n.m. & 2.67 & $71 \%$ \\
\hline $12-17$ & n.m. & n.m. & n.m. & n.m. & n.m. & n.m. \\
\hline Mean & $1.46^{*}$ & & 1.41 & & 1.46 & \\
\hline
\end{tabular}

Mean shear wave velocities (SWV) and coefficient of variation (CV; standard deviation/mean in \%) of different insertion depths, probes and frequencies. ${ }^{*}=$ mean includes misfiring with less than twelve, but six or more successful measurements. N.m. = measurement failed 
- Table 3 'General' vs.'penetration' preset.

\begin{tabular}{|c|c|c|c|c|}
\hline & \multicolumn{2}{|c|}{ General } & \multicolumn{2}{|c|}{ Penetration } \\
\hline & SWV & CV & SWV & CV \\
\hline $\begin{array}{l}\text { Position and size } \\
\text { B-mode ROI }\end{array}$ & $(\mathrm{m} / \mathrm{s})$ & & $(\mathrm{m} / \mathrm{s})$ & \\
\hline $\begin{array}{l}\text { Left upper square } \\
1 \mathrm{~cm} \times 1 \mathrm{~cm}\end{array}$ & 5.13 & $4 \%$ & $5.29^{\circ}$ & $2 \%$ \\
\hline $\begin{array}{l}\text { Left lower square } \\
1 \mathrm{~cm} \times 1 \mathrm{~cm}\end{array}$ & 3.69 & $8 \%$ & $4.11^{\circ}$ & $4 \%$ \\
\hline $\begin{array}{l}\text { Right upper square } \\
1 \mathrm{~cm} \times 1 \mathrm{~cm}\end{array}$ & 4.91 & $3 \%$ & 4.96 & $3 \%$ \\
\hline $\begin{array}{l}\text { Right lower square } \\
1 \mathrm{~cm} \times 1 \mathrm{~cm}\end{array}$ & 3.94 & $7 \%$ & $4.61^{\circ}$ & $5 \%$ \\
\hline $\begin{array}{l}\text { Left-sided rectangle } \\
1 \mathrm{~cm} \times 2 \mathrm{~cm}\end{array}$ & 4.66 & $4 \%$ & 4.70 & $2 \%$ \\
\hline $\begin{array}{l}\text { Right-sided } \\
\text { rectangle } \\
1 \mathrm{~cm} \times 2 \mathrm{~cm}\end{array}$ & 4.92 & $3 \%$ & 5.01 & $2 \%$ \\
\hline $\begin{array}{l}\text { Upper rectangle } \\
2 \mathrm{~cm} \times 1 \mathrm{~cm}\end{array}$ & 6.8 & $4 \%$ & $7.08^{\circ}$ & $1 \%$ \\
\hline $\begin{array}{l}\text { Lower rectangle } \\
2 \mathrm{~cm} \times 1 \mathrm{~cm}\end{array}$ & 4.92 & $3 \%$ & $5.61^{\circ}$ & $3 \%$ \\
\hline $\begin{array}{l}\text { One large square } \\
2 \mathrm{~cm} \times 2 \mathrm{~cm}\end{array}$ & 6.52 & $2 \%$ & 6.51 & $1 \%$ \\
\hline
\end{tabular}

Different B-mode ROI positions and sizes ( $\vee$ Fig. 2) showing the dependency of SWV and coefficient of variation (CV) to B-mode insertion depth and width of muscle measured with the $9 \mathrm{~L}$ linear probe. $S W V=$ mean of twelve single $S W V$ measurements. ${ }^{\circ}=$ significantly increased SWV using the 'penetration' mode ing scanning depth and if there was bone below the ROI. They used a linear probe for shallow tissue regions and a convex probe for deeper areas, but did not made a subgroup analysis of convex versus linear probe results. In line with this, increasing the B-mode ROI size from $1 \mathrm{~cm}$ to $2 \mathrm{~cm}$ in the vertical direction and using the linear probe led to a decrease in the SWVmean of the entire SWVmap. Practically speaking, the influence of ROI placement depth may be relativized by increasing the $\mathrm{ROI}$ size in the vertical direction.

In contrast to ARFI, the size of the B-mode ROI as well as the circular ROI can be defined manually for 2D-SWE by the sonographer. $\mathrm{B}$-mode ROI size is the area of pulse deposition and SWV measurement. Increasing the B-mode ROI width significantly increased the SWVmean in our study. The exact reason is unclear to us. There may be some tissue displacement tracking algorithm limitation for fast SWV values measured in a narrow B-mode ROI width. On the other hand, increasing the height of the ROI extends the integration height of the shear-wave front, which filters out the effect of tissue heterogeneity and probably provides more accurate estimates of the average tissue behavior.

Increasing the circular ROI size from the center of the B-mode ROI showed little effect on the SWVmean in our measurements. SWVmean is the sum of all SWV values of each pixel within the ROI divided by the number of pixels within the ROI. This is in accordance with other studies reporting on non-focal diseases [18, 19]. Schellhaas et al. [18] found that the variation of circular ROI size from 5 to $20 \mathrm{~mm}$ seems to be of minor importance in cirrhotic patients and healthy individuals. Bortolotto et al. [20] did not find significant differences in the muscle stiffness of healthy subjects for different circular ROI sizes. In contrast to diffuse organ diseases, circular ROI size does play a role in focal disease, e. g. breast lesions, working with SWVmean cut-off values for malignancy [21, 22]. In this case increasing the ROI size will lead to a lower SWVmean.

Using the manufacturer preset 'penetration' caused an increase in SWVmean and a decrease in CV, especially for the ROls with a deeper placement depth. According to the manufacturer, this mode

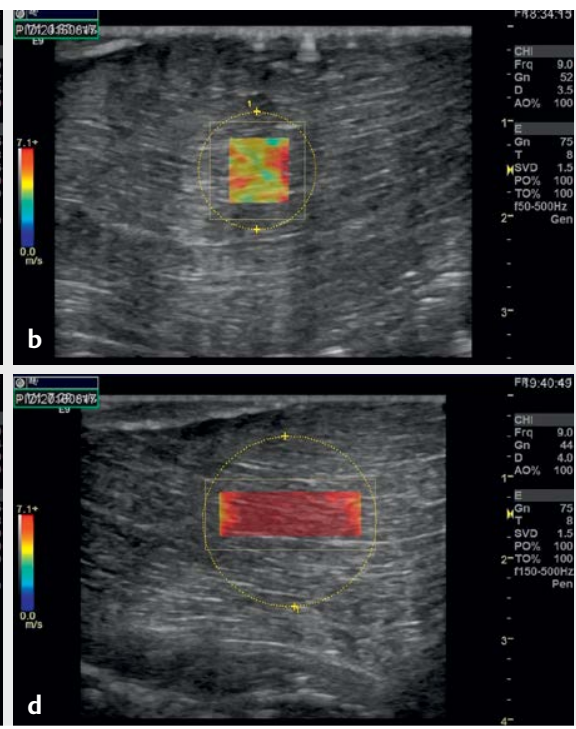

Fig. 3 2D SWE of porcine muscle: a B-mode image with $1 \mathrm{~cm} \times 1 \mathrm{~cm}$ B-mode ROI and SWV overlapped, $\mathbf{b}$ circular ROI placement, $\mathbf{c} 1 \mathrm{~cm}$ width and $2 \mathrm{~cm}$ depth B-mode ROI shows decreasing SWV measurements and values with increasing depth and $\mathbf{d} 2 \mathrm{~cm}$ width and $1 \mathrm{~cm}$ depth B-mode ROI at the same placement depth showing markedly increased SWV values with increasing ROI width. 


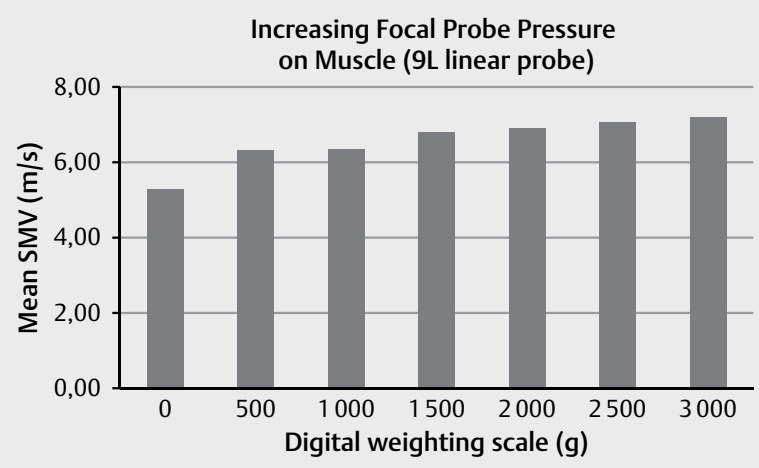

- Fig. 4 Compression force dependency of SWV values applied on porcine muscle and measured in longitudinal fibre direction at $1 \mathrm{~cm}$ insertion depth.

optimizes the ultrasound sequence (e.g., transmit frequency) to penetrate deeper tissues. Therefore, we think that it is advisable to use the manufacturer preset 'penetration' with the drawback of a lower SWE frame rate for muscle elastography with the linear probe. The image processing algorithms used to compute SWVmean are specific to the ultrasound system [23].

Gennisson et al. [24] found that shear waves propagate much faster along beef muscle fibers, as compared to perpendicularly or any interval of rotation therein. Later investigations support these initial findings, as longitudinal transducer orientations obtained the most reliable measures of muscle elasticity $[25,26]$. Cortez et al. [27] observed that SWV values in muscle measured in the longitudinal direction were significantly higher on average than in the cross-section direction and showed lower CVs (6.9\% to $12.5 \%)$ than the latter (CV 12.6\% to 15.6\%). This is in accordance with our measurements with decreased SWVmean and increased CV for the cross-section of the muscle compared to the longitudinal direction using the linear probe. The structure of muscle is heterogeneous and anisotropic, which leads to a strong distortion of the shear wave front as it propagates forward in the tissue. In particular, muscle behaves like a fiber-reinforced composite; the SWV is faster along the muscle fiber bundles than in the amorphous muscle background. Depending on the exact geometry of the fiber bundles, the SWV varies.

For soft tissue imaging, probe compression should be considered. Kot et al. [28] reported a significant increase in the maximum and mean muscle SWV with an increase in focal linear probe pressure evaluated by a combination of subjective and visual assessment. Soft tissues are strongly non-linear, with elastography measurements correlating to the derivative of the stress-strain curve at a specific operation point. Although non-linearity is generally considered to be a cofounding factor, it has not yet fully exploited potential for tissue differentiation and disease diagnosis, if well characterized [29-31]. We quantified the effect of probe pressure on SWV and found a significant increase with pressure most pronounced between 0 and $500 \mathrm{~g}$ (corresponding to 0-9.8 kPa). Because high pressure may not be acceptable for patient comfort, minimal compression should be used to remain just in contact with the body surface.
The majority of ultrasound systems do not allow access to raw data and are closed systems with respect to post-processing. Very recently, academies, clinics and vendors began taking first standardization steps to quantitatively calibrate SWV systems with respect to common reference materials [29].

Our limitations are that we performed ex vivo measurements. Therefore, the real SWVmean values, likewise in a certified muscle elasticity phantom, are not known. The effects of arterial and venous pressure, body versus room temperature, dynamic muscle elasticity, a layer of cutis and subcutis between probe and muscle, as well as animal versus human may alter the influence of confounders. For living tissue, the muscle stiffness strongly increases in the longitudinal direction when the living muscle supports increased weight, whereas the difference in the cross-section direction is less significant. Therefore, in a non-excited state as in ex vivo the SWVmean in the longitudinal and cross-sectional directions become more similar [25]. Any long delay in the preparation and measurement of ex vivo samples could alter shear wave measurements as muscle will decompose over time [32].

In conclusion, 2D-SWE of ex vivo muscle is influenced by $\mathrm{B}$-mode ROI placement depth and size, preset mode, muscle fiber orientation, as well as probe choice, frequency and pressure. As shown by the results of this study, it is advisable to assess possible confounders of ultrasound elastography before application in clinical musculoskeletal research studies and routine patient assessment, as well as using a standardized approach about confounders.

\section{Compliance with ethical standards}

\section{Ethics approval}

All procedures were carried out in accordance with the 1964 Declaration of Helsinki and its later amendments or comparable ethical standards.

\section{Conflicts of Interest}

The authors declare that they have no conflicts of interest.

\section{References}

[1] Ricci P, Marigliano C, Cantisani V et al. Ultrasound evaluation of liver fibrosis: Preliminary experience with acoustic structure quantification (ASQ) software. La Radiologia medica 2013; 118: 995-1010

[2] Correas JM, Drakonakis E, Isidori AM et al. Update on ultrasound elastography: Miscellanea. Prostate, testicle, musculo-skeletal. Eur ] Radiol 2013; 82: 1904-1912

[3] Dietrich CF, Barr RG, Farrokh A et al. Strain Elastography-How To Do It? Ultrasound International Open 2017; 3: E137-e149

[4] Brandenburg JE, Eby SF, Song P et al. Ultrasound elastography: The new frontier in direct measurement of muscle stiffness. Arch Phys Med Rehabil 2014; 95: 2207-2219

[5] Ewertsen C, Carlsen JF, Christiansen IR et al. Evaluation of healthy muscle tissue by strain and shear wave elastography-Dependency on depth and ROI position in relation to underlying bone. Ultrasonics 2016; 71: 127-133 
[6] Sporea I, Gradinaru-Tascau O, Bota S et al. How many measurements are needed for liver stiffness assessment by 2D-Shear Wave Elastography (2D-SWE) and which value should be used: The mean or median? Med Ultrason 2013; 15: 268-272

[7] Tang A, Cloutier G, Szeverenyi NM et al. Ultrasound Elastography and MR Elastography for Assessing Liver Fibrosis: Part 2, Diagnostic Performance, Confounders, and Future Directions. AJR Am J Roentgenol 2015; 205: 33-40

[8] Park MS, Kim SW, Yoon KT et al. Factors Influencing the Diagnostic Accuracy of Acoustic Radiation Force Impulse Elastography in Patients with Chronic Hepatitis B. Gut Liver 2016; 10: 275-282

[9] Pfeifer L, Goertz RS, Neurath MF et al. Comparison of Acoustic Radiation Force Impulse (ARFI) Elastography Measurements with the 4C1 Transducer to the 6C1HD Transducer: A Phantom and Patient Study. Ultraschall Med 2016, doi:10.1055/s-0041-107974

[10] Chang S, Kim MJ, Kim J et al. Variability of shear wave velocity using different frequencies in acoustic radiation force impulse (ARFI) elastography: A phantom and normal liver study. Ultraschall Med 2013; 34: 260-265

[11] Yang L, Yuan J, Wang Q et al. Reliability analysis of acoustic radiation force impulse ultrasound imaging with virtual touch tissue quantification: Ex vivo ox liver. Ultrasound Q 2015; 31: 59-62

[12] Song P, Macdonald M, Behler R et al. Two-dimensional shear-wave elastography on conventional ultrasound scanners with time-aligned sequential tracking (TAST) and comb-push ultrasound shear elastography (CUSE). IEEE Trans Ultrason Ferroelectr Freq Control 2015; 62: 290-302

[13] Palmeri ML, McAleavey SA, Fong KL et al. Dynamic mechanical response of elastic spherical inclusions to impulsive acoustic radiation force excitation. IEEE Trans Ultrason Ferroelectr Freq Control 2006; 53 : 2065-2079

[14] Varbobitis IC, Siakavellas SI, Koutsounas IS et al. Reliability and applicability of two-dimensional shear-wave elastography for the evaluation of liver stiffness. Eur J Gastroenterol Hepatol 2016, doi:10.1097/meg.0000000000000686

[15] Boursier J, Zarski JP, de Ledinghen $V$ et al. Determination of reliability criteria for liver stiffness evaluation by transient elastography. Hepatology 2013; 57: 1182-1191

[16] Procopet B, Berzigotti A, Abraldes JG et al. Real-time shear-wave elastography: Applicability, reliability and accuracy for clinically significant portal hypertension. J Hepatol 2015; 62: 1068-1075

[17] Thiele M, Madsen BS, Procopet B et al. Reliability Criteria for Liver Stiffness Measurements with Real-Time 2D Shear Wave Elastography in Different Clinical Scenarios of Chronic Liver Disease. Ultraschall Med 2016, doi:10.1055/s-0042-108431

[18] Schellhaas B, Strobel D, Wildner D et al. Two-dimensional shear-wave elastography: A new method comparable to acoustic radiation force impulse imaging? Eur J Gastroenterol Hepatol 2017; 29: 723-729
[19] Huang Z, Zheng J, Zeng J et al. Normal liver stiffness in healthy adults assessed by real-time shear wave elastography and factors that influence this method. Ultrasound Med Biol 2014; 40: 2549-2555

[20] Bortolotto C, Lungarotti L, Fiorina I et al. Influence of subjects' characteristics and technical variables on muscle stiffness measured by shear wave elastosonography. J Ultrasound 2017; 20: 139-146

[21] Blank MAB, Antaki JF. Breast lesion elastography region of interest selection and quantitative heterogeneity: A systematic review and meta-analysis. Ultrasound Med Biol 2017; 43: 387-397

[22] Skerl K, Vinnicombe S, Giannotti E et al. Influence of region of interest size and ultrasound lesion size on the performance of 2D shear wave elastography (SWE) in solid breast masses. Clin Radiol 2015; 70 : 1421-1427

[23] Drakonaki EE, Allen GM, Wilson DJ. Ultrasound elastography for musculoskeletal applications. Br J Radiol 2012; 85: 1435-1445

[24] Gennisson JL, Catheline S, Chaffai S et al. Transient elastography in anisotropic medium: Application to the measurement of slow and fast shear wave speeds in muscles. J Acoust Soc Am 2003; 114: 536-541

[25] Gennisson JL, Deffieux T, Mace E et al. Viscoelastic and anisotropic mechanical properties of in vivo muscle tissue assessed by supersonic shear imaging. Ultrasound Med Biol 2010; 36: 789-801

[26] Eby SF, Song P, Chen S et al. Validation of shear wave elastography in skeletal muscle. J Biomech 2013; 46: 2381-2387

[27] Cortez CD, Hermitte L, Ramain A et al. Ultrasound shear wave velocity in skeletal muscle: A reproducibility study. Diagn Interv Imaging 2016; 97: 71-79

[28] Kot BC, Zhang Z], Lee AW et al. Elastic modulus of muscle and tendon with shear wave ultrasound elastography: Variations with different technical settings. PloS one 2012; 7: e44348

[29] Shiina T, Nightingale KR, Palmeri ML et al. WFUMB guidelines and recommendations for clinical use of ultrasound elastography: Part 1: basic principles and terminology. Ultrasound Med Biol 2015; 41: 1126-1147

[30] Jiang Y, Li GY, Qian LX et al. Characterization of the nonlinear elastic properties of soft tissues using the supersonic shear imaging (SSI) technique: Inverse method, ex vivo and in vivo experiments. Med Image Anal 2015; 20: 97-111

[31] Sanabria SJRM, Otesteanu CF, Mazza E, Goksel O. Non-linear characterization of the liver by combining shear and longitudinal wave speed with strain observations. In Int Tissue Elasticity Conf (ITEC). Vermont, NE, USA: 2016

[32] Parker KJ, Partin A, Rubens DJ. What do we know about shear wave dispersion in normal and steatotic livers? Ultrasound Med Biol 2015; 41: 1481-1487 